\title{
Supplementary for An amino acid-swapped genetic code
}

Tomoshige Fujino ${ }^{1}$, Masahiro Tozaki ${ }^{1}$, and Hiroshi Murakami ${ }^{1,2, k}$

${ }^{1}$ Department of Biomolecular Engineering, Graduate School of Engineering, Nagoya University, Nagoya, 464-8603, Japan

2 Institute of Nano-Life-Systems, Institutes of Innovation for Future Society, Nagoya University, Nagoya, 464-8603, Japan

* To whom correspondence should be addressed. Tel: +81-52-789-3327; Fax: +81-52-789-3327; Email: murah@chembio.nagoya-u.ac.jp 
Concentrations of aaRSs and EF-Tu/Ts normalized against those of the initial translation system.

\begin{tabular}{|c|c|c|c|c|c|c|c|c|c|c|c|c|c|}
\hline \multirow{5}{*}{$\begin{array}{l}\frac{\sigma}{3} \\
\frac{0}{3} \\
\frac{0}{0}\end{array}$} & AlaRS & 1 & 3.5 & 1 & 1 & 1 & 1 & 1 & 1 & 1 & 1 & 1 & 1 \\
\hline & ArgRS & 1 & 6 & 1 & 1 & 1 & 1 & 1 & 1 & 1 & 1 & 1 & 1 \\
\hline & ValRS & 1 & 6 & 1 & 1 & 1 & 1 & 1 & 1 & 1 & 1 & 1 & 1 \\
\hline & AspRS & 1 & 6 & 1 & 1 & 1 & 1 & 1 & 1 & 1 & 1 & 1 & 1 \\
\hline & CysRS & 1 & 6 & 1 & 1 & 1 & 1 & 1 & 1 & 1 & 1 & 1 & 1 \\
\hline \multirow{5}{*}{$\begin{array}{l}N \\
\stackrel{2}{3} \\
\frac{0}{0}\end{array}$} & GlnRS & 1 & 1 & 6 & 1 & 1 & 6 & 1 & 1 & 1 & 1 & 1 & 1 \\
\hline & GluRS & 1 & 1 & 6 & 1 & 1 & 1 & 6 & 1 & 1 & 1 & 6 & 6 \\
\hline & HisRS & 1 & 1 & 6 & 1 & 1 & 1 & 1 & 6 & 1 & 1 & 1 & 1 \\
\hline & lleRS & 1 & 1 & 3.5 & 1 & 1 & 1 & 1 & 1 & 3.5 & 1 & 3.5 & 3.5 \\
\hline & TyrRS & 1 & 1 & 6 & 1 & 1 & 1 & 1 & 1 & 1 & 6 & 1 & 1 \\
\hline \multirow{5}{*}{$\begin{array}{l}\text { m } \\
\text { 음 } \\
\text { 임 }\end{array}$} & LeuRS & 1 & 1 & 1 & 6 & 1 & 1 & 1 & 1 & 1 & 1 & 1 & 1 \\
\hline & LysRS & 1 & 1 & 1 & 6 & 1 & 1 & 1 & 1 & 1 & 1 & 1 & 1 \\
\hline & MetRS & 1 & 1 & 1 & 6 & 1 & 1 & 1 & 1 & 1 & 1 & 1 & 1 \\
\hline & PheRS & 1 & 1 & 1 & 3.5 & 1 & 1 & 1 & 1 & 1 & 1 & 1 & 1 \\
\hline & TrpRS & 1 & 1 & 1 & 6 & 1 & 1 & 1 & 1 & 1 & 1 & 1 & 1 \\
\hline \multirow{6}{*}{$\begin{array}{l}\text { ナ } \\
\text { ○ } \\
\text { 이 } \\
\text { ㄴ }\end{array}$} & SerRS & 1 & 1 & 1 & 1 & 6 & 1 & 1 & 1 & 1 & 1 & 1 & 1 \\
\hline & ThrRS & 1 & 1 & 1 & 1 & 6 & 1 & 1 & 1 & 1 & 1 & 1 & 1 \\
\hline & AsnRS & 1 & 1 & 1 & 1 & 6 & 1 & 1 & 1 & 1 & 1 & 1 & 1 \\
\hline & GlyRS & 1 & 1 & 1 & 1 & 6 & 1 & 1 & 1 & 1 & 1 & 1 & 1 \\
\hline & ProRS & 1 & 1 & 1 & 1 & 6 & 1 & 1 & 1 & 1 & 1 & 1 & 1 \\
\hline & EF-Tu/Ts & 1 & 1 & 1 & 1 & 1 & 1 & 1 & 1 & 1 & 1 & 1 & 3.5 \\
\hline
\end{tabular}

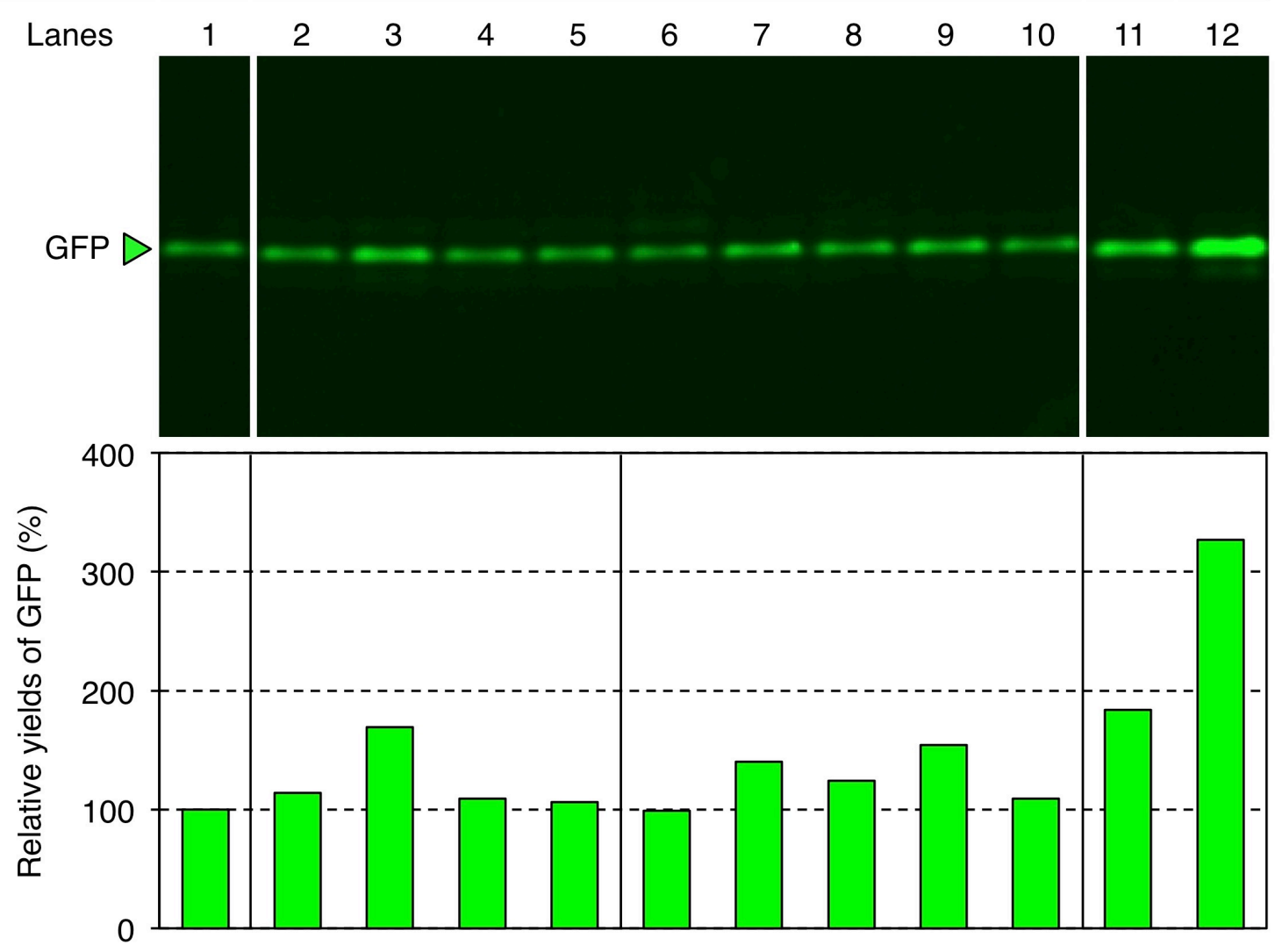

Supplementary Figure 1. Optimization of aaRSs and EF-Tu/Ts concentrations in the reconstituted cell-free translation system carrying T7-tRNAs. The concentrations of aaRSs and EF-Tu/Ts in each translation system were shown in the table above the gel (concentrations increased to 6 -fold or 3.5- 
fold were highlighted in orange). GFP mRNA encoded by the mini-standard (mini-Std) genetic code was added to the translation systems and the yields of the functional GFP were measured by using native-PAGE analysis. The yields of GFP were normalized against that produced in the initial cell-free translation system (lane 1). Results: We first investigated effects of increased aaRS(s) concentration on the yield of GFP. We divided 20 aaRSs into four groups (group 1: AlaRS, ArgRS, ValRS, AspRS, and CysRS, group 2: GlnRS, GluRS, HisRS, IleRS, and TyrRS, group 3: LeuRS, LysRS, MetRS, PheRS, and TrpRS, group 4: SerRS, ThrRS, AsnRS, GlyRS, and ProRS) and prepared four translation systems in which the concentrations of each group of aaRSs were increased to 6-fold (the concentration of AlaRS, IleRS, and PheRS were increased to 3.5-fold to avoid addition of large volume of aaRS mixture into the translation system). Among the four translation systems, the one with additional group 2 aaRSs gave 1.7 times increased yield of GFP (lane 3). We then increased the concentration of each aaRS in group 2, and found that increased concentration of GluRS or lleRS improved the yields of GFP (lane 7: 1.4 times and lane 9: 1.5 times respectively). We also found that combination of increased GluRS and IleRS improved GFP yield to 1.8 times (lane 11), which is similar to the result of the addition of ARS group 2 (lane 4: 1.7 times). Finally, we tested the effect of increased concentration of EF-Tu/Ts on the yield of GFP. We found that the 3.5-fold increase of the concentration of EF-Tu/Ts in the translation system further improved GFP yield by about $80 \%$ (lane 12). Through the optimization of both aaRSs and EF-Tu/Ts concentrations, the yield of GFP produced by the reconstituted cell-free translation system carrying T7-tRNAs improved to 3.3 times. 

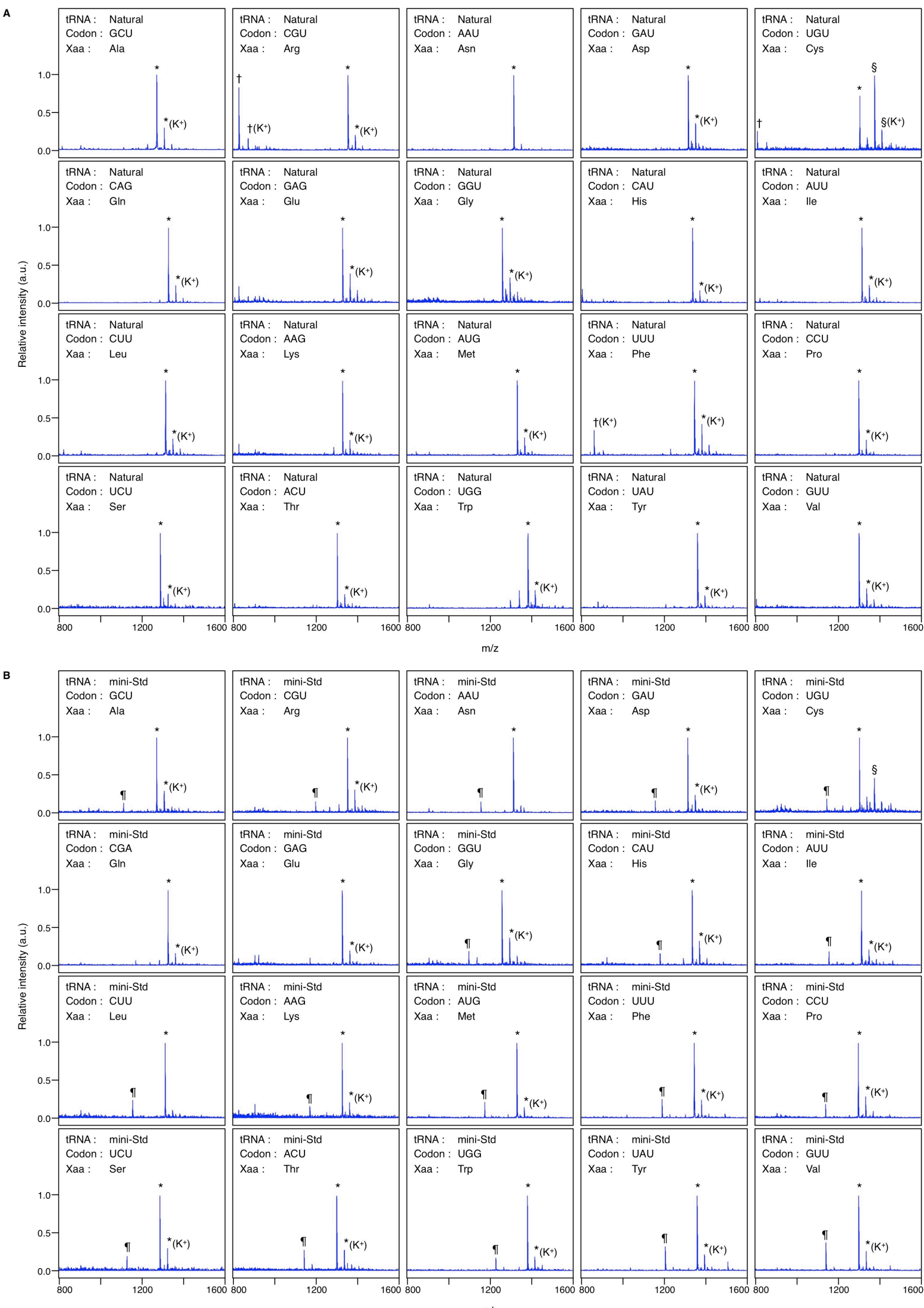

Supplementary Figure 2. MS analysis of model peptides synthesized in cell-free translation systems containing natural tRNAs (A) or the mini-Std tRNA set (B). The peaks are labeled as follows: 
full-length model peptide containing a desired AA in the position Xaa $\left(^{*}\right)$, truncated peptide lacking the sequence after Xaa (fMet-Tyr-Tyr-Tyr-Xaa) (†), full-length model peptide containing $\beta$ mercaptoethanol-bound Cys (§), and truncated peptide lacking fMet (Tyr-Tyr-Tyr-Xaa-Asp-Asp-ArgAsp) (đ). The calculated and observed masses of the peptides are shown in Table S2. 


\section{A Streptavidin}

mini-Std CTAGTAATACGACTCACTATAGgGTTAACTTTAAGAAgGAGATATACATATGGATCCCTC 60

SL CTAGTAATACGACTCACTATAGgGTTAACTTTAAGAAGGAGATATACATATGGATCCCCT 60

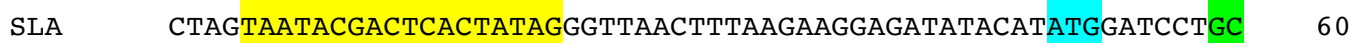

mini-Std TAAGGACTCCAAGGCTCAGGTTTCCGCTGCCGAGGCTGGCATTACCGGCACTTGGTATAA 120

$\begin{array}{lll}\mathrm{SL} & \text { TAAGGACCTCAAGGCCCAGGTTCTTGCCGCTGAGGCTGGTATTACTGGCACCTGGTACAA } & 120\end{array}$

SLA CAAGGATGCTAAGCTTCAGGTTGCCCTTCTTGAGCTCGGCATTACTGGCACCTGGTACAA 120

mini-Std CCAGCTTGGCTCCACCTTTATTGTCACCGCTGGCGCTGATGGTGCTCTTACTGGCACCTA 180

SL CCAGTCTGGCCTCACTTTCATCGTCACTGCTGGTGCCGATGGCGCTTCTACCGGTACCTA 180

SLA CCAGTCTGGTGCCACCTTCATCGTCACTCTTGGTCTTGATGGCCTCTCTACCGGCACCTA 180

mini-Std TGAGTCTGCCGTTGGCAATGCTGAGTCCCGTTATGTTCTCACTGGTCGCTATGACTCCGC 240

SL TGAGCTTGCCGTTGGCAATGCTGAGCTCCGCTATGTCTCCACCGGTCGTTATGACCTCGC 240

SLA TGAGGCCCTTGTTGGCAATCTCGAGGCTCGCTATGTCTCCACTGGTCGTTATGACGCTCT 240

mini-Std TCCTGCTACTGACGGTTCTGGTACTGCTCTTGGTTGGACCGTTGCCTGGAAGAACAATTA 300

SL TCCTGCTACTGACGGTCTTGGCACTGCTTCTGGCTGGACTGTCGCTTGGAAGAACAACTA 300

SLA TCCCCTCACTGATGGTGCTGGTACCCTTTCTGGCTGGACTGTCCTCTGGAAGAACAACTA 300

mini-Std TCGTAACGCCCATTCCGCCACCACTTGGTCTGGTCAGTATGTTGGTGGTGCTGAGGCTCG 360

SL CCGCAATGCCCATCTTGCCACCACTTGGCTTGGTCAGTACGTTGGTGGTGCTGAGGCCCG 360

SLA CCGCAATCTCCATGCCCTTACCACTTGGGCTGGTCAGTACGTTGGCGGTCTTGAGCTCCG 360

mini-Std TATCAATACTCAGTGGCTTCTCACCTCCGGCACTACCGAGGCTAACGCCTGGAAGTCTAC 420

SL TATCAACACCCAGTGGTCCTCCACTCTCGGCACCACTGAGGCCAATGCCTGGAAGCTCAC 420

SLA TATCAACACTCAGTGGTCCTCCACTGCTGGTACTACTGAGCTCAATCTCTGGAAGGCTAC 420

mini-Std CCTTGTTGGTCACGACACTTTCACTAAGGTCAAGCCCTCCGCCGCCTCTATCGATGCTGC 480

SL TTCCGTTGGTCACGACACCTTTACCAAGGTCAAGCCTCTTGCTGCCCTCATTGATGCTGC 480

SLA CTCCGTTGGTCACGACACCTTTACCAAGGTCAAGCCTGCCCTTCTCGCCATTGACCTCCT 480

mini-Std TAAGAAGGCTGGCGTCAATAACGgTAACCCTCTTGATGCCGTCCAGCAGTAACTATGT 538

SL CAAGAAGGCTGGCGTCAACAATGGCAATCCCTCTGATGCCGTTCAGCAGTAACTATGT 538

SLA CAAGAAGCTTGGCGTTAACAATGGCAATCCCTCTGACCTTGTCCAGCAGTAACTATGT 


\section{B GFP}

mini-Std GGCGTAATACGACTCACTATAGGATTAACTTTAACAAGGAGAAAAACATGAATTCTAAGG SL GGCGTAATACGACTCACTATAGGATTAACTTTAACAAGGAGAAAAACATGAATCTTAAGG SLA GGCGTAATACGACTCACTATAGGATTAACTTTAACAAGGAGAAAAACATGAATGCTAAGG

mini-Std GTGAGGAGCTTTTCACTGGTGTTGTTCCTATCCTTGTTGAGCTTGATGGTGATGTTAACG SL GTGAGGAGTCTTTCACTGGTGTTGTTCCTATCTCTGTTGAGTCTGATGGTGATGTTAACG SLA GTGAGGAGTCTTTCACTGGTGTTGTTCCTATCTCTGTTGAGTCTGATGGTGATGTTAACG

mini-Std GTCATAAGTTCTCTGTTTCTGGTGAGGGTGAGGGTGATGCTACTTACGGTAAGCTTACTC SL GTCATAAGTTCCTTGTTCTTGGTGAGGGTGAGGGTGATGCTACTTACGGTAAGTCTACTT

mini-Std TTAAGTTCATCTGTACTACTGGTAAGCTTCCTGTTCCTTGGCCTACTCTTGTTACTACTT

CTAAGTTCATCTGTACTACTGGTAAGTCTCCTGTTCCTTGGCCTACTTCTGTTACTACI SLA CTAAGTTCATCTGTACTACTGGTAAGTCTCCTGTTCCTTGGCCTACTTCTGTTACTACTT

mini-Std TCTCTTACGGTGTTCAGTGTTTCTCTCGTTACCCTGATCATATGAAGCGTCATGATTTCT SL TCCTTTACGGTGTTCAGTGTTTCCTTCGTTACCCTGATCATATGAAGCGTCATGATTTCT

mini-Std TCAAGTCTGCTATGCCTGAGGGTTACGTTCAGGAGCGTACTATCTCTTTCAAGGATGATG

TCAAGCTTGCTATGCCTGAGGGTTACGTTCAGGAGCGTACTATCCTTTTCAAGGATGATG

mini-Std GTAACTACAAGACTCGTGCTGAGGTTAAGTTCGAGGGTGATACTCTTGTTAACCGTATCG SL GTAACTACAAGACTCGTGCTGAGGTTAAGTTCGAGGGTGATACTTCTGTTAACCGTATCG SLA GTAACTACAAGACTCGTCTTGAGGTTAAGTTCGAGGGTGATACTTCTGTTAACCGTATCG

mini-Std AGCTTAAGGGTATCGATTTCAAGGAGGATGGTAACATCCTTGGTCATAAGCTTGAGTACA SL AGTCTAAGGGTATCGATTTCAAGGAGGATGGTAACATCTCTGGTCATAAGTCTGAGTACA SLA AgtCTAAgGgtATCGATTTCAAGGAgGATGGTAACATCTCTGGTCATAAGTCTGAGTACA

mini-Std ACTACAACTCTCATAACGTTTACATCACTGCTGATAAGCAGAAGAACGGTATCAAGGCTA SL ACTACAACCTTCATAACGTTTACATCACTGCTGATAAGCAGAAGAACGGTATCAAGGCTA

mini-Std ACTTCAAGATCCGTCATAACATCGAGGATGGTTCTGTTCAGCTTGCTGATCATTACCAGC SL ACTTCAAGATCCGTCATAACATCGAGGATGGTCTTGTTCAGTCTGCTGATCATTACCAGC SLA ACTTCAAGATCCGTCATAACATCGAGGATGGTGCTGTTCAGTCTCTTGATCATTACCAGC

mini-Std AGAACACTCCTATCGGTGATGGTCCTGTTCTTCTTCCTGATAACCATTACCTTTCTACTC SL AGAACACTCCTATCGGTGATGGTCCTGTTTCTTCTCCTGATAACCATTACTCTCTTACTC SLA AGAACACTCCTATCGGTGATGGTCCTGTTTCTTCTCCTGATAACCATTACTCTGCTACTC

mini-Std AGTCTGCTCTTTCTAAGGATCCTAACGAGAAGCGTGATCATATGGTTCTTCTTGAGTTCG

SL AGCTTGCTTCTCTTAAGGATCCTAACGAGAAGCGTGATCATATGGTTTCTTCTGAGTTCG

mini-Std TTACTGCTGCTGGTATCACTCATGGTATGGATGAGCTCTACAAGTAAGCATACTGGATTC

SL TTACTGCTGCTGGTATCACTCATGGTATGGATGAGTCCTACAAGTAAGCATACTGGATTC 


\begin{tabular}{|c|c|c|c|c|c|c|c|c|c|c|}
\hline \multirow{2}{*}{\multicolumn{2}{|c|}{$\begin{array}{c}\text { tRNA sets } \\
\text { Genes }\end{array}$}} & \multicolumn{3}{|c|}{ Natural or mini-Std } & \multicolumn{3}{|c|}{ SL } & \multicolumn{3}{|c|}{ SLA } \\
\hline & & mini-Std & SL & SLA & mini-Std & SL & SLA & mini-Std & SL & SLA \\
\hline \multirow{3}{*}{ Number of residues } & Ser & 14 & 8 & 8 & 8 & 14 & 25 & 25 & 25 & 14 \\
\hline & Leu & 8 & 14 & 25 & 14 & 8 & 8 & 14 & 8 & 8 \\
\hline & Ala & 25 & 25 & 14 & 25 & 25 & 14 & 8 & 14 & 25 \\
\hline \multicolumn{2}{|c|}{ Molecular weights } & 16622 & 16778 & 17240 & 16778 & 16622 & 16798 & 17050 & 16798 & 16622 \\
\hline \multicolumn{2}{|c|}{ Difference of M.W. from mini-Std streptavidin } & 0 & +156 & +618 & +156 & 0 & +176 & +428 & +176 & \\
\hline
\end{tabular}

\begin{tabular}{|c|c|c|c|c|c|c|c|c|c|c|}
\hline \multirow{2}{*}{\multicolumn{2}{|c|}{$\frac{\text { tRNA sets }}{\text { Genes }}$}} & \multicolumn{3}{|c|}{ Natural or mini-Std } & \multicolumn{3}{|c|}{ SL } & \multicolumn{3}{|c|}{ SLA } \\
\hline & & mini-Std & SL & SLA & mini-Std & SL & SLA & mini-Std & SL & SLA \\
\hline \multirow{3}{*}{ Number of residues } & Ser & 12 & 19 & 19 & 19 & 12 & 9 & 9 & 9 & 12 \\
\hline & Leu & 19 & 12 & 9 & 12 & 19 & 19 & 12 & 19 & 19 \\
\hline & Ala & 9 & 9 & 12 & 9 & $\mathbf{9}$ & 12 & 19 & 12 & 9 \\
\hline \multicolumn{2}{|c|}{ Molecular weights } & 26910 & 26728 & 26602 & 26728 & 26910 & 26862 & 26568 & 26862 & 26910 \\
\hline \multicolumn{2}{|c|}{ Difference of M.W. from mini-Std GFP } & 0 & -182 & -308 & -182 & 0 & -48 & -342 & -48 & 0 \\
\hline
\end{tabular}

Supplementary Figure 3. Sequences of template DNAs encoding model proteins and the translation products. Sequences of template DNAs encoding streptavidin (A) or GFP (B). The sequences were encoded by the mini-Std, SL, or SLA genetic codes. The T7 promoter is highlighted in yellow. Start and stop codons are highlighted in blue. AA-swapped codons are highlighted in green. (C) The number of Ser, Leu, and Ala residues and the molecular weights of translation products synthesized in cell-free translation systems with correct and incorrect combinations of tRNA sets and genetic codes. 

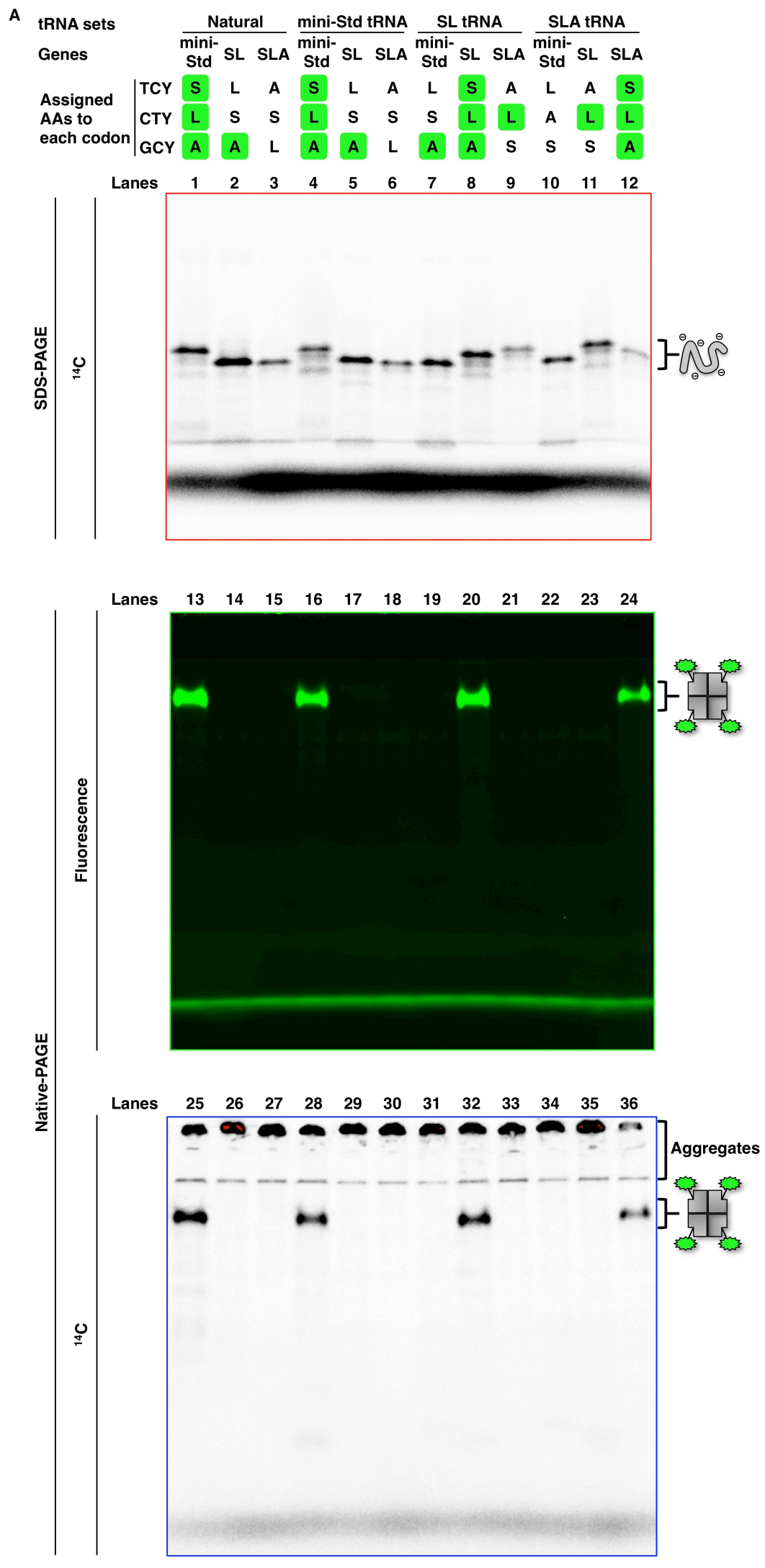

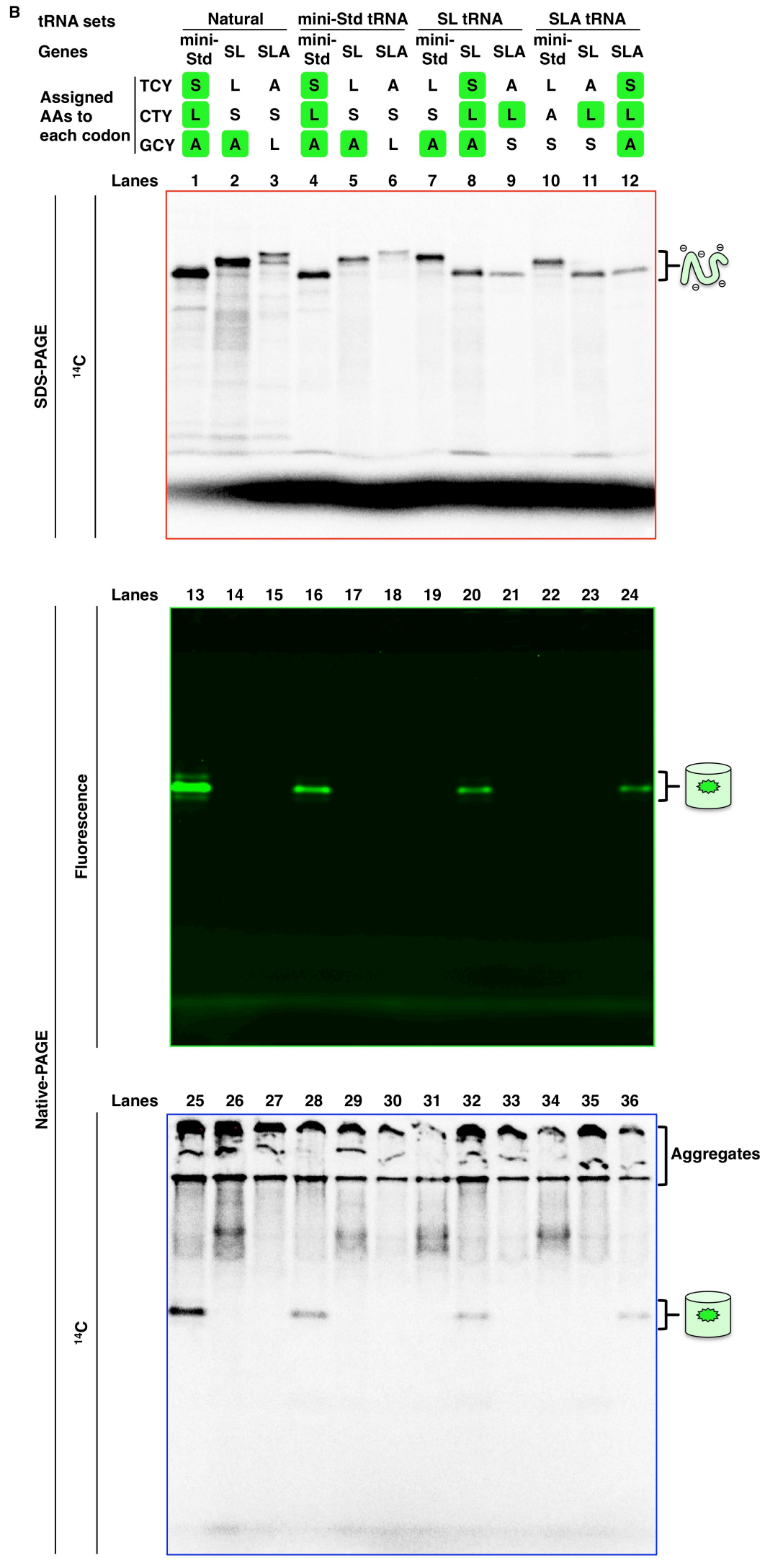

Supplementary Figure 4. Full image of the gels shown in Figure 4. Details are described in Figure 4. 


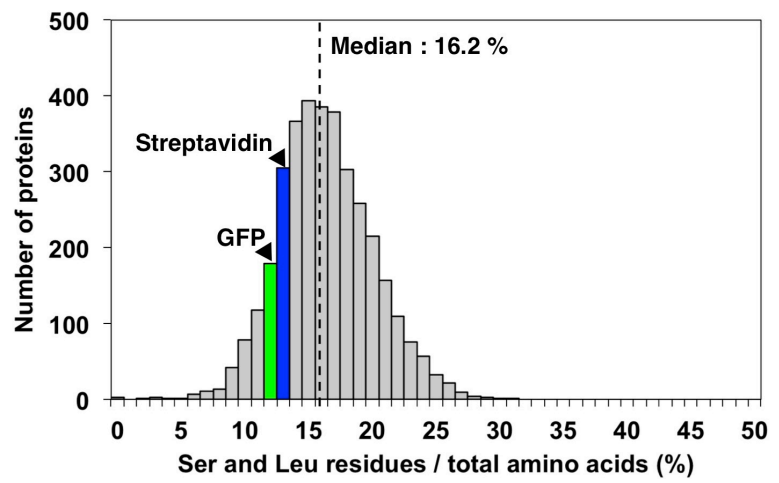

B

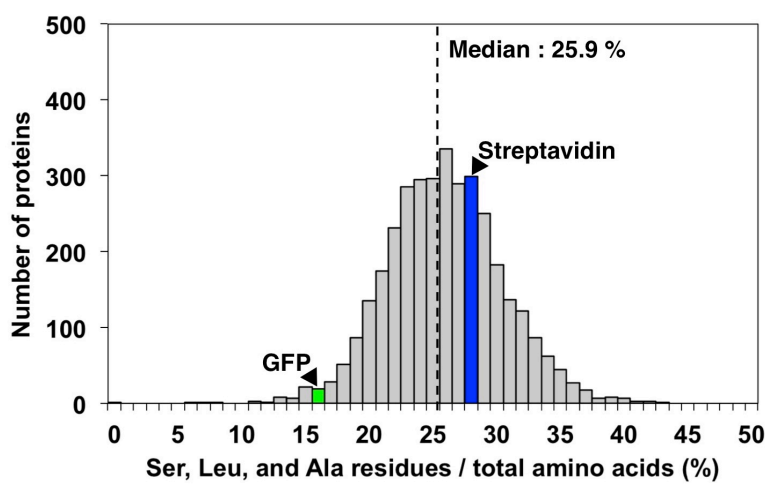

Supplementary Figure 5. Fraction of Ser and Leu (A) or Ser, Leu, and Ala residues (B) over all AA residues in each protein encoded in 3547 CDSs of E. coli MDS42. Fraction of Ser and Leu residues or Ser, Leu, and Ala residues were calculated from the protein sequences of 3547 CDSs of E. coli MDS42 strain. Bars corresponding to the fraction of model proteins were highlighted in blue (streptavidin) and green (GFP). 
A
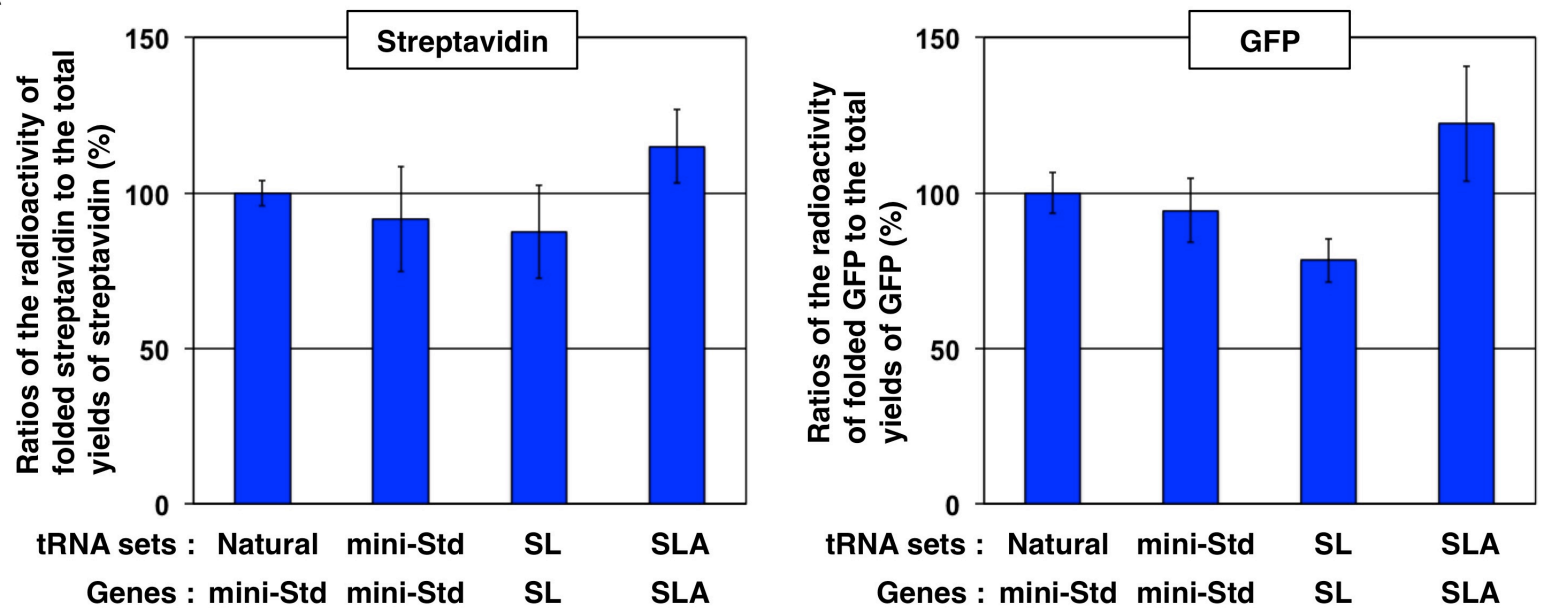

B
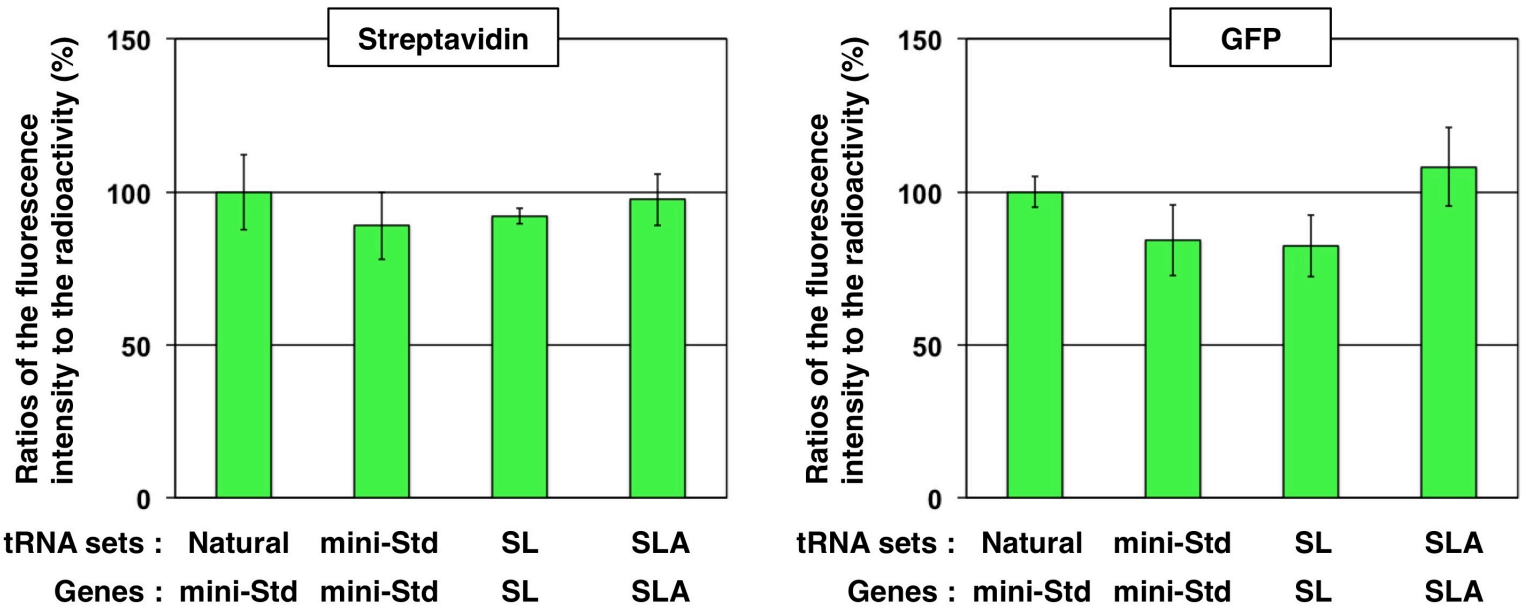

Supplementary Figure 6. Folding efficiency and fluorescence activity of proteins synthesized with the correct combination of tRNA sets and genes. (A) Ratios of the radioactivity of folded proteins determined by native PAGE to the total yields of proteins determined by SDS-PAGE. The ratios were calculated from the samples synthesized in cell-free translation systems with correct combinations of tRNA sets and genes. (B) Ratios of the fluorescence intensity to the radioactivity observed in fluorescence imaging and autoradiography of the gel in native PAGE. Error bars show the standard deviation of each experiment in triplicate. 
Supplementary Table 1. Sequences of T7-tRNAs and primer sets used to prepare T7-tRNA templates.

\begin{tabular}{|c|c|c|c|c|c|c|c|c|c|}
\hline \multirow{2}{*}{ Names } & \multirow{2}{*}{ Anticodons } & \multirow{2}{*}{ Length } & \multirow{2}{*}{ tRNA sequences ( $5^{\prime}$ to $3^{\prime}$ ) } & \multicolumn{2}{|c|}{ Extensin primers } & \multicolumn{2}{|c|}{ 1st PCR primers } & \multicolumn{2}{|r|}{ 2nd PCR primers } \\
\hline & & & & Fw. primers & Rv. primers & Fw. primers & Rv. primers & \begin{tabular}{|l|} 
Fw. primers \\
\end{tabular} & Rv. primers \\
\hline tRNA ${ }^{\text {Ala }}$ & GGC & 76 & $\begin{array}{l}\text { GGGGC UAUAG CUCAG CUGGG } \\
\text { AGAGC GCUUG CAUGG CAUGC } \\
\text { AAGAG GUUAG CGGUU CGAUC } \\
\text { CCGCU UAGCU CCACC A }\end{array}$ & Ala2 (GGC)-5.F49 & Ala2 (GGC)at.R43 & T7ex5.F22 & Ala2 (GGC)-3.R38 & T7ex5.F22 & Ala2 (GGC)-3.R20 \\
\hline $\operatorname{tRNA}^{\mathrm{Ala}}{ }_{\mathrm{GAC}}$ & GAG & 76 & $\begin{array}{l}\text { GGGGC UAUAG CUCAG CUGGG } \\
\text { AGAGC GCUUG CUUGA GGUGG } \\
\text { AAGAG GUUAG CGGUU CGAUC } \\
\text { CCGCU UAGCU CCACC A }\end{array}$ & Ala1 (UGC)-5.F49 & nAla1L (UGC->GAG)at.R43 & T7ex5.F22 & Ala1 (UGC)-3.R38 & T7ex5.F22 & Ala1 (UGC)-3.R20 \\
\hline tRNA $A^{a r g}$ & GCG & 77 & $\begin{array}{l}\text { GCAUC CGUAG CUCAG CUGGA } \\
\text { UAGAG UACUC GGCUG CGAAC } \\
\text { CGAGC GGUCG GAGG UCGAA } \\
\text { UCCUC CCGGA UGCAC CA }\end{array}$ & Arg2 (ACG->GCG)-5.F50 & Arg2 (ACG->GCG)at.R43 & T7ex5.F22 & Arg2 (ACG $>$ GCG)-3.R38 & T7ex5.F22 & Arg2 (ACG->GCG)-3.R20 \\
\hline tRNA Asn & GUU & 76 & $\begin{array}{l}\text { GCCUC UGUAG UUCAG UCGGU } \\
\text { AGAAC GGCGG ACUGU UAAUC } \\
\text { CGUAU GUVAC UGGUU CGAGU } \\
\text { CCAGU CAGAG GCGCC A }\end{array}$ & Asn 1G72C (GTT)-5.F49 & Asn 1G72C (GTT)at.R43 & T7ex5.F22 & Asn 1G72C (GTT)-3.R38 & T7ex5.F22 & Asn 1G72C (GTT)-3.R20 \\
\hline tRNA Asp & GuC & 77 & $\begin{array}{l}\text { GGAGC GGUAG UUCAG UCGGU } \\
\text { UAGAA UACCU GCCUG UCACG } \\
\text { CAGGG GGUCG CGGGGU UCGAG } \\
\text { UCCCG UCCGU UCCGC CA }\end{array}$ & Asp (GTC)-5.F50 & Asp (GTC)at.R43 & T7ex5.F22 & Asp (GTC)-3.R38 & T7ex5.F22 & Asp (GTC)-3.R20 \\
\hline $\operatorname{tRNA}^{\mathrm{cys}}$ & GCA & 74 & $\begin{array}{l}\text { GGGGC GUUAA CAAAG CGGUU } \\
\text { AUGUA GCGGA UUGCA AAUCC } \\
\text { GUCUA GUCCG GUUCG ACUCC } \\
\text { GGAAC GCGCC UCCA }\end{array}$ & Cys (GCA)-5.F48 & Cys (GCA)at.R43 & T7ex5.F22 & Cys (GCA)-3.R37 & T7ex5.F22 & Cys (GCA)-3.R20 \\
\hline tRNA $A^{\text {Glu }}$ & cuc & 76 & $\begin{array}{l}\text { GUCCC CUUCG UCUAG AGGCC } \\
\text { CAGGA CACCG CCCUC UCACG } \\
\text { GCGGU AACAGG GGGUU CGAAU } \\
\text { CCCCU AGGGG ACGCC A }\end{array}$ & Glu (TTC->CTC)-5.F50 & Glu (TTC->CTC)at.R43 & T7ex5.F22 & Glu (TTC->CTC)-3.R37 & T7ex5.F22 & Glu (TTC->CTC)-3.R20 \\
\hline $\operatorname{tRNA}^{\mathrm{aly}}$ & GCC & 76 & $\begin{array}{l}\text { GCGGG AAUAG CUCAG UUGGU } \\
\text { AGAGC ACGAC CUUGC CAAGG } \\
\text { UCGGG GUCGC GAGUU CGAGU } \\
\text { CUCGU UUCCC GCUCC A }\end{array}$ & Gly3 (GCC)-5.F49 & Gly3 (GCC)at.R43 & T7ex5.F22 & Gly3 (GCC)-3.R38 & T7ex5.F22 & Gly3 (GCC)-3.R2O \\
\hline tRNA ${ }^{\text {His }}$ & GUG & 77 & $\begin{array}{l}\text { GGUGG CUAUA GCUCA GUUGG } \\
\text { UAGAG CCCUGG GAUUG UGAUU } \\
\text { CCAGU UGUUG UGGGU UCGAA } \\
\text { UCCCA UUAGC CACCC CA }\end{array}$ & His (GTG)-5.F50 & His (GTG)at.R43 & T7ex5.F22 & His (GTG)-3.R38 & T7ex5.F22 & His (GTG)-3.R2O \\
\hline tRNA & GAU & 77 & $\begin{array}{l}\text { GGGCU UGUAG CUCAG GUGGU } \\
\text { UAGAG CGCAC CCCUG AUAAG } \\
\text { GGUGA GGUCG GUGG UUCAAG } \\
\text { UCCAC UCAGG CCCAC CA }\end{array}$ & Ile (GAT) 1G72C-5.F50 & Ille 1G72C (GAT)at.R43 & T7ex5.F22 & Ile 1G72C(GAT)-3.R38 & T7ex5.F22 & IIle 1G72C(GAT)-3.R20 \\
\hline tRNA $^{\text {Leu }}$ & GAG & 87 & $\begin{array}{l}\text { GCCGA GGUGG UGGAA UUGGU } \\
\text { AGACA CGCUA CCUUG AGGGG } \\
\text { GUAGU GCCCA AUAGG GCUUA } \\
\text { CGGGU UCAAG UCAGG UCCUC } \\
\text { GGUAC CA }\end{array}$ & Leu2 (GAG)-5.F50 & Leu2 (GAG)at.R43 & T7ex5.F22 & Leu2 (GAG)-3.R48 & T7ex5.F22 & Leu2 (GAG)-3.R20 \\
\hline tRNA ${ }^{\text {Lew }}{ }_{G G A}$ & GGA & 87 & $\begin{array}{l}\text { GCCGA GGUGG UGGAA UUGGU } \\
\text { AGACA CGCUA CCCUG GAAAG } \\
\text { GUAGU GCCCA AUAGG GCUUA } \\
\text { CGGGU GCAAAG UCCCG UCCUC } \\
\text { GGUAC CA }\end{array}$ & Leu2 (GAG)-5.F50 & nLeu2S (GAG->GGA)at.R43 & T7ex5.F22 & Leu2 (GAG)-3.R48 & T7ex5.F22 & Leu2 (GAG)-3.R20 \\
\hline $\operatorname{tRNA}^{2 / s}$ & cuu & 76 & $\begin{array}{l}\text { GGGUC GUUAG CUCAG UUGGU } \\
\text { AGAGC AGUUG ACUCU UAAUC } \\
\text { AAUUG GUUGC AGGUU CGAAU } \\
\text { CCUGC ACGAC CCACC A }\end{array}$ & Lys (TTT->CTT)-5.F49 & Lys (TTT->CTT)at. R43 & T7ex5.F22 & Lys (TTT->CTT)-3.R38 & T7ex5.F22 & Lys (TTT->CTT)-3.R20 \\
\hline tRNA $A^{\text {met }}$ & CAU & 77 & $\begin{array}{l}\text { GGCGG GGUGG AGCAG CCUGG } \\
\text { UAGCU CGUCG GGCUC AUAAC } \\
\text { CCGAA GAUCG UCGGU UCAAA } \\
\text { UCCGG CCCCC GCAAC CA }\end{array}$ & fMet 1G (CAT)-5.F50 & fMet 1G (CAT)at.R43 & T7ex5.F22 & fMet 1G (CAT)-3.R38 & T7ex5.F22 & fMet 1G (CAT)-3.R20 \\
\hline tRNA ${ }^{\text {mot }}$ & CAU & 76 & $\begin{array}{l}\text { GGCUA CGUAG CUCAG UUGGU } \\
\text { UAGAG CACAU CACUC AUAAU } \\
\text { GAUGG GGUCA CAGGU UCGAA } \\
\text { UCCCG UCGUA GCCAC CA }\end{array}$ & Met (CAT)-5.F50 & Met (CAT)at.R43 & T7ex5.F22 & Met (CAT)-3.R38 & T7ex5.F22 & Met (CAT)-3.R20 \\
\hline tRNA $^{\text {phe }}$ & GAA & 76 & $\begin{array}{l}\text { GCCCG GAUAG CUCAG UCGGU } \\
\text { AGAGC AGGGG ACUGA AAAUC } \\
\text { CCCGU GUCCCU UGGUU CGAUU } \\
\text { CCGAG UCCGG GCACC A }\end{array}$ & Phe 32C (GAA)-5.F49 & Phe $32 \mathrm{C}$ (GAA)at.R43 & T7ex5.F22 & Phe 32C (GAA)-3.R38 & T7ex5.F22 & Phe 32C (GAA)-3.R20 \\
\hline tRNA ${ }^{\text {Setro }}{ }_{\text {GAG }}$ & GAG & 90 & $\begin{array}{l}\text { GGAGA GAUGC CGGAG CGGCU } \\
\text { GAACG GAACCG GUUUG AGGUA } \\
\text { CCGGA GUAGG GGCAA CUCUA } \\
\text { CCGGG GGUUC AAAUC CCCCU } \\
\text { CUCUC CGCCA }\end{array}$ & Ser2 (CGA)-5.F50 & nSer2L (CGA->GAG)at.R43 & T7ex5.F22 & Ser2 (CGA)-3.R51 & T7ex5.F22 & Ser2 (CGA)-3.R20 \\
\hline tRNA ${ }^{\text {ser }}{ }_{G \rightarrow C}$ & GGC & 90 & $\begin{array}{l}\text { GGAGA GAUGC CGGAG CGGCU } \\
\text { GAACG GACCCG GUAUG GCAUA } \\
\text { CCGGA GUAGG GGCAA CUCUA } \\
\text { CCGGG GGUUC AAAUC CCCCU } \\
\text { CUCUC CGCCA }\end{array}$ & Ser2 (CGA)-5.F50 & nSer2A (CGA->GGC)at.R43 & T7ex5.F22 & Ser2 (CGA)-3.R51 & T7ex5.F22 & Ser2 (CGA)-3.R20 \\
\hline tRNA $A^{\text {ser }}$ & GGA & 88 & $\begin{array}{l}\text { GGUGA GGUGU CCGAG UGGCU } \\
\text { GAAGG AGGACC GCCUG GAAAG } \\
\text { UGUGU AUACG GCAAC GUAUC } \\
\text { GGGGG UUCGA AUCCC CCCCU } \\
\text { CACCG CCA }\end{array}$ & Ser5 (GGA)-5.F50 & Ser5 (GGA)at.R43 & T7ex5.F22 & Ser5 (GGA)-3.R49 & T7ex5.F22 & Ser5 (GGA)-3.R20 \\
\hline $\operatorname{tRNA}^{\mathrm{Tn} \mathrm{T}}$ & GGU & 76 & $\begin{array}{l}\text { GCUGA UAUAG CUCAG UUGGU } \\
\text { AGAGC GCACC CUUGG UAAGG } \\
\text { GUGAG GUCGG CAGUU CGAAU } \\
\text { CUGCC UAUCA GCACC A }\end{array}$ & Thr3 (GGT)-5.F49 & Thr3 (GGT)at.R43 & T7ex5.F22 & Thr3 (GGT)-3.R38 & T7ex5.F22 & Thr3 (GGT)-3.R20 \\
\hline tRNA $A^{T \times r}$ & GUA & 85 & $\begin{array}{l}\text { GGUGG GGUUC CCGAG CGGCC } \\
\text { AAAGG GAGCA GACUG UAAAU } \\
\text { CUGCC GUUCAC AGACU UCGAA } \\
\text { GGUUC GAAUC CUUCC CCCAC } \\
\text { CACCA }\end{array}$ & Tyr (GTA)-5.F50 & Tyr (GTA)at.R43 & T7ex5.F22 & Tyr (GTA)-3.R46 & T7ex5.F22 & Tyr (GTA)-3.R20 \\
\hline tRNA $^{\text {val }}$ & GAC & 77 & $\begin{array}{l}\text { GCGUC CGUAG CUCAG UUGGU } \\
\text { UAGAG CACCAA CCUUG ACAUG } \\
\text { GUGGG GGUCG GUGGUUCGAG } \\
\text { UCCAC UCGGA CGCAC CA }\end{array}$ & Val2 (GAC)-5.F50 & Val2 (GAC)at.R43 & T7ex5.F22 & Val2 (GAC)-3.R38 & T7ex5.F22 & Val2 (GAC)-3.R20 \\
\hline tRNA $A^{\sin }$ & CUG & 75 & $\begin{array}{l}\text { UGGGG UAUCG CCAAG CGGUA } \\
\text { AGGCA CCGGA UUCUG AUUCC } \\
\text { GGCAU UCCGAA GGUUC GAAUC } \\
\text { CUCGU ACCCC AGCCA }\end{array}$ & GIn2 (CTG)-5I .F49 & Gln2 (CTG)at.R43 & T7pro-leader.F36 & Gln2 (CTG)-3.R38 & T7ex5.F22 & Gln2 (CTG)-3.R20 \\
\hline tRNA $A^{\text {pro }}$ & GGG & 77 & $\begin{array}{l}\text { CGGGCA CGUAG CGCAG CCUGG } \\
\text { UAGCG CACCG UCAUG GGGUG } \\
\text { UCGGG GGUCG GAGGU UCAAA } \\
\text { UCCUC UCGUG CCGAC CA }\end{array}$ & Pro1 (GGG)-51.F50 & Pro1-2 (GGG)at.R43 & T7pro-leader.F36 & Pro1 (GGG)-3.R38 & T7ex5.F22 & Pro1 (GGG)-3.R20 \\
\hline tRNA $^{\text {Tip }}$ & CCA & 76 & $\begin{array}{l}\text { AGGGG CGUAG UUCAA UUGGU } \\
\text { AGAGC ACCGG UCUCC AAAAC } \\
\text { CGGGU GUUGG GAGUU CGAGU } \\
\text { CUCUC CGCCC CUGCC A }\end{array}$ & $\operatorname{Trp}(\mathrm{CCA})-51 . \mathrm{F} 49$ & $\operatorname{Trp}(\mathrm{CCA})$ at.R43 & T7pro-leader.F36 & Trp (CCA)-3.R38 & T7ex5.F22 & Trp (CCA)-3.R20 \\
\hline
\end{tabular}


Supplementary Table 2. The calculated and observed masses of the peptides. ${ }^{a}$

\begin{tabular}{|c|c|c|c|c|c|c|c|c|c|c|}
\hline \multirow{3}{*}{$\begin{array}{l}\text { tRMA } \\
\text { sets }\end{array}$} & \multirow{3}{*}{ Codons } & \multirow{3}{*}{ Xaas } & \multicolumn{2}{|c|}{ Full-length peptides $\left(^{*}\right)$} & \multicolumn{2}{|c|}{ Addition of $\beta$-mercaptoethanol ( ()} & \multicolumn{2}{|c|}{ Truncated peptide $(\dagger)$} & \multicolumn{2}{|c|}{ Truncated peptide (I) } \\
\hline & & & fMet-Tyr-Tyr-Tyr- & Asp-Asp-Arg-Asp & fMet-Tyr-Tyr-Tyr-X & Asp-Asp-Arg-Asp & fMet-Tyr- & r-Tyr-Xaa & Tyr-Tyr-Tyr-Xaa- & sp-Asp-Arg-Asp \\
\hline & & & Calculated MS & Observed MS & Calculated MS & Observed MS & Calculated MS & Observed MS & Calculated MS & Observed MS \\
\hline Natural & GCU & Ala & 1239.45 & 1239.34 & & & & & & \\
\hline Natural & CGU & $\operatorname{Arg}$ & 1324.52 & 1324.42 & & & 823.34 & 823.38 & & \\
\hline Natural & AAU & Asn & 1282.46 & 1282.48 & & & & & & \\
\hline Natural & GAU & Asp & 1283.44 & 1283.63 & & & & & & \\
\hline Natural & UGU & Cys & 1271.42 & 1271.26 & 1347.43 & 1347.32 & $808.2(+K)$ & 808.06 & & \\
\hline Natural & CAG & Gln & 1296.47 & 1296.48 & & & & & & \\
\hline Natural & GAG & Glu & 1297.46 & 1297.54 & & & & & & \\
\hline Natural & GGU & Gly & 1225.44 & 1225.66 & & & & & & \\
\hline Natural & CAU & His & 1305.47 & 1305.63 & & & & & & \\
\hline Natural & AUU & lle & 1281.50 & 1281.81 & & & & & & \\
\hline Natural & CUU & Leu & 1281.50 & 1281.31 & & & & & & \\
\hline Natural & AAG & Lys & 1296.51 & 1296.29 & & & & & & \\
\hline Natural & AUG & Met & 1299.46 & 1299.36 & & & & & & \\
\hline Natural & UUU & Phe & 1315.48 & 1315.37 & & & $852.26(+\mathrm{K})$ & 852.34 & & \\
\hline Natural & $\mathrm{CCU}$ & Pro & 1265.47 & 1265.51 & & & & & & \\
\hline Natural & UCU & Ser & 1255.45 & 1255.69 & & & & & & \\
\hline Natural & ACU & Thr & 1269.46 & 1270.22 & & & & & & \\
\hline Natural & UGG & Trp & 1354.49 & 1354.53 & & & & & & \\
\hline Natural & UAU & Tyr & 1331.48 & 1331.60 & & & & & & \\
\hline Natural & GUU & Val & 1267.48 & 1267.67 & & & & & & \\
\hline mini-Std & GCU & Ala & 1239.45 & 1239.34 & & & & & 1080.42 & 1080.26 \\
\hline mini-Std & CGU & $\operatorname{Arg}$ & 1324.52 & 1324.56 & & & & & 1165.49 & 1166.46 \\
\hline mini-Std & AAU & Asn & 1282.46 & 1282.54 & & & & & 1123.43 & 1123.51 \\
\hline mini-Std & GAU & Asp & 1283.44 & 1283.66 & & & & & 1124.41 & 1124.55 \\
\hline mini-Std & UGU & Cys & 1271.42 & 1271.72 & 1347.43 & 1347.71 & & & 1112.39 & 1112.65 \\
\hline mini-Std & CAG & Gln & 1296.47 & 1296.26 & & & & & & \\
\hline mini-Std & GAG & Glu & 1297.46 & 1297.44 & & & & & & \\
\hline mini-Std & GGU & Gly & 1225.44 & 1225.47 & & & & & 1066.41 & 1066.42 \\
\hline mini-Std & CAU & His & 1305.47 & 1305.69 & & & & & 1146.44 & 1146.63 \\
\hline mini-Std & AUU & Ile & 1281.50 & 1281.41 & & & & & 1122.47 & 1122.41 \\
\hline mini-Std & CUU & Leu & 1281.50 & 1281.47 & & & & & 1122.47 & 1122.41 \\
\hline mini-Std & AAG & Lys & 1296.51 & 1296.51 & & & & & 1137.48 & 1137.46 \\
\hline mini-Std & AUG & Met & 1299.46 & 1299.62 & & & & & 1140.43 & 1140.63 \\
\hline mini-Std & UUU & Phe & 1315.48 & 1315.74 & & & & & 1156.45 & 1156.70 \\
\hline mini-Std & CCU & Pro & 1265.47 & 1265.17 & & & & & 1106.44 & 1106.21 \\
\hline mini-Std & UCU & Ser & 1255.45 & 1255.20 & & & & & 1096.42 & 1096.19 \\
\hline mini-Std & ACU & Thr & 1269.46 & 1269.31 & & & & & 1110.43 & 1110.28 \\
\hline mini-Std & UGG & Trp & 1354.49 & 1354.31 & & & & & 1195.46 & 1195.27 \\
\hline mini-Std & UAU & Tyr & 1331.48 & 1331.34 & & & & & 1172.45 & 1172.40 \\
\hline mini-Std & GUU & Val & 1267.48 & 1267.35 & & & & & 1108.45 & 1108.32 \\
\hline SL & UCU & Leu & 1281.50 & 1281.44 & & & & & 1122.47 & 1122.46 \\
\hline SL & CUU & Ser & 1255.45 & 1255.52 & & & & & 1096.42 & 1096.47 \\
\hline ASL & GCU & Ser & 1255.45 & 1255.48 & & & & & 1096.42 & 1096.45 \\
\hline ASL & UCU & Leu & 1281.50 & 1281.69 & & & & & 1122.47 & 1122.60 \\
\hline ASL & CUU & Ala & 1239.45 & 1239.65 & & & & & 1080.42 & 1080.58 \\
\hline
\end{tabular}

a The peaks are labeled as follows: full-length model peptides containing a desired AA in the position Xaa $\left({ }^{*}\right)$, fulllength model peptides containing $\beta$-mercaptoethanol-bound Cys $(\S)$, truncated peptides lacking the sequence after Xaa (fMet-Tyr-Tyr-Tyr-Xaa) (†), and truncated peptides lacking fMet (Tyr-Tyr-Tyr-Xaa-Asp-Asp-Arg-Asp) ( $(\mathbb{T})$. 
Supplementary Table 3. Primer sequences used in this study. ${ }^{\text {a }}$

\begin{tabular}{|c|c|c|}
\hline & Primer names & Sequences $\left(5^{\prime}\right.$ to $\left.3^{\prime}\right)$ \\
\hline \multirow{22}{*}{ 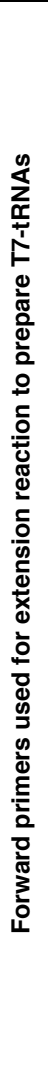 } & fMet 1G (CAT)-5.F50 & GTAATACGACTCACTATAGGCGGGGTGGAGCAGCCTGGTAGCTCGTCGGG \\
\hline & Ala2 (GGC)-5.F49 & GTAATACGACTCACTATAGGGGCTATAGCTCAGCTGGGAGAGCGCTTGC \\
\hline & Arg2 (ACG->GCG)-5.F50 & GTAATACGACTCACTATAGCATCCGTAGCTCAGCTGGATAGAGTACTCGG \\
\hline & Asn 1G72C (GTT)-5.F49 & GTAATACGACTCACTATAGCCTCTGTAGTTCAGTCGGTAGAACGGCGGA \\
\hline & Asp (GTC)-5.F50 & GTAATACGACTCACTATAGGAGCGGTAGTTCAGTCGGTTAGAATACCTGC \\
\hline & Cys (GCA)-5.F48 & GTAATACGACTCACTATAGGCGCGTTAACAAAGCGGTTATGTAGCGGA \\
\hline & Gln2 (CTG)-5I .F49 & GGAACGCGCGACTCTAATTGGGGTATCGCCAAGCGGTAAGGCACCGGA \\
\hline & Glu (TTC->CTC)-5.F50 & GTAATACGACTCACTATAGTCCCCTTCGTCTAGAGGCCCAGGACACCGCC \\
\hline & Gly3 (GCC)-5.F49 & GTAATACGACTCACTATAGCGGGAATAGCTCAGTTGGTAGAGCACGACC \\
\hline & His (GTG)-5.F50 & GTAATACGACTCACTATAGGTGGCTATAGCTCAGTTGGTAGAGCCCTGGA \\
\hline & Ile (GAT) 1G72C-5.F50 & GTAATACGACTCACTATAGGGCTTGTAGCTCAGGTGGTTAGAGCGCACCC \\
\hline & Leu2 (GAG)-5.F50 & GTAATACGACTCACTATAGCCGAGGTGGTGGAATTGGTAGACACGCTACC \\
\hline & Lys (TTT->CTT)-5.F49 & GTAATACGACTCACTATAGGGTCGTTAGCTCAGTTGGTAGAGCAGTTGA \\
\hline & Met (CAT)-5.F50 & GTAATACGACTCACTATAGGCTACGTAGCTCAGTTGGTTAGAGCACATCA \\
\hline & Phe 32C (GAA)-5.F49 & GTAATACGACTCACTATAGCCCGGATAGCTCAGTCGGTAGAGCAGGGGA \\
\hline & Pro2 (GGG)-5I.F50 & GGAACGCGCGACTCTAATCGGCACGTAGCGCAGCCTGGTAGCGCACCGTC \\
\hline & Ser5 (GGA)-5.F50 & GTAATACGACTCACTATAGGTGAGGTGTCCGAGTGGCTGAAGGAGCACGC \\
\hline & Thr3 (GGT)-5.F49 & GTAATACGACTCACTATAGCTGATATAGCTCAGTTGGTAGAGCGCACCC \\
\hline & Trp (CCA) -5I.F49 & GGAACGCGCGACTCTAATAGGGGCGTAGTTCAATTGGTAGAGCACCGGT \\
\hline & Tyr (GTA)-5.F50 & GTAATACGACTCACTATAGGTGGGGTTCCCGAGCGGCCAAAGGGAGCAGA \\
\hline & Val2 (GAC)-5.F50 & GTAATACGACTCACTATAGCGTCCGTAGCTCAGTTGGTTAGAGCACCACC \\
\hline & Ala1 (UGC)-5.F49 & GTAATACGACTCACTATAGGGGCTATAGCTCAGCTGGGAGAGCGCCTGC \\
\hline \multirow{25}{*}{ 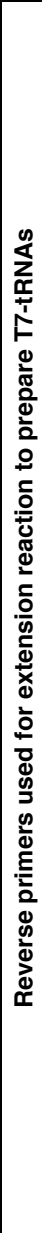 } & fMet 1G (CAT)at.R43 & GAACCGACGATCTTCGGGTTATGAGCCCGACGAGCTACCAGGC \\
\hline & Ala2 (GGC)at.R43 & GAACCGCTGACCTCTTGCATGCCATGCAAGCGCTCTCCСАGCT \\
\hline & Arg2 (ACG->GCG)at.R43 & GAACCTCCGACCGCTCGGTTCGCAGCCGAGTACTCTATCCAGC \\
\hline & Asn 1G72C (GTT)at.R43 & GAACCAGTGACATACGGATTAACAGTCCGCCGTTCTACCGACT \\
\hline & Asp (GTC)at.R43 & GAACCCGCGACCCCCTGCGTGACAGGCAGGTATTCTAACCGAC \\
\hline & Cys (GCA)at.R43 & CGAACCGGACTAGACGGATTTTGCAATCCGCTACATAACCGCTT \\
\hline & GIn2 (CTG)at.R43 & GAACCTCGGAATGCCGGAATCAGAATCCGGTGCCTTACCGCTT \\
\hline & Glu (TTC->CTC)at.R43 & CGAACCCCTGTTACCGCCGTGAGAGGGCGGTGTCCTGGGCCTC \\
\hline & Gly3 (GCC)at.R43 & GAACTCGCGACCCCGACCTTGGCAAGGTCGTGCTCTACCAACT \\
\hline & His (GTG)at.R43 & GAACCCACGACAACTGGAATCACAATCCAGGGCTCTACCAACT \\
\hline & Ile 1G72C (GAT)at.R43 & GAACCACCGACCTCACCCTTATCAGGGGTGCGCTCTAACCACC \\
\hline & Leu2 (GAG)at.R43 & GCCCTATTGGGCACTACCACCTCAAGGTAGCGTGTCTACCAAT \\
\hline & Lys (TTT->CTT)at.R43 & GAACCTGCGACCAATTGATTAAGAGTCAACTGCTCTACCAACT \\
\hline & Met (CAT)at.R43 & GAACCTGTGACCCCATCATTATGAGTGATGTGCTCTAACCAAC \\
\hline & Phe 32C (GAA)at.R43 & GAACCAAGGACACGGGGATTTTCAGTCCCCTGCTCTACCGACT \\
\hline & Pro2 (GGG)at.R43 & GAACCTCCGACCCCCGACACCCCATGACGGTGCGCTACCAGGC \\
\hline & Ser5 (GGA)at.R43 & ACGTTGCCGTATACACACTTTCCAGGCGTGCTCCTTCAGCCAC \\
\hline & Thr3 (GGT)at.R43 & GAACTGCCGACCTCACCCTTACCAAGGGTGCGCTCTACCAACT \\
\hline & $\operatorname{Trp}$ (CCA)at.R43 & GAACTCCCAACACCCGGTTTTGGAGACCGGTGCTCTACCAATT \\
\hline & Tyr (GTA)at.R43 & GAAGTCTGTGACGGCAGATTTACAGTCTGCTCCCTTTGGCCGC \\
\hline & Val2 (GAC)at.R43 & GAACCACCGACCCCCACCATGTCAAGGTGGTGCTCTAACCAAC \\
\hline & nLeu2S (GAG->GGA)at.R43 & GCCCTATTGGGCACTACCTTTCGAGGGTAGCGTGTCTACCAAT \\
\hline & nSer2L (CGA->GAG)at.R43 & AGTTGCCCCTACTCCGGTACCTCAAACCGGTCCGTTCAGCCGC \\
\hline & nAla2L (GGC->GAG)at.R43 & GAACCGCTGACCTCTTGCACCTCAAGCAAGCGCTCTCCCAGCT \\
\hline & nSer2A (CGA->GGC)at.R43 & AGTTGCCCCTACTCCGGTATGCCATACCGGTCCGTTCAGCCGC \\
\hline
\end{tabular}


Supplementary Table 3 (continued)

\begin{tabular}{|c|c|c|}
\hline & fMet 1G (CAT)-3.R38 & TGGTTGCGGGGGCCGGATTTGAACCGACGATCTTCGGG \\
\hline & Ala2 (GGC)-3.R38 & TGGTGGAGCTAAGCGGGATCGAACCGCTGACCTCTTGC \\
\hline & Arg2 (ACG->GCG)-3.R38 & TGGTGCATCCGGGAGGATTCGAACCTCCGACCGCTCGG \\
\hline & Asn 1G72C (GTT)-3.R38 & TGGCGCCTCTGACTGGACTCGAACCAGTGACATACGGA \\
\hline & Asp (GTC)-3.R38 & TGGCGGAACGGACGGGACTCGAACCCGCGACCCCCTGC \\
\hline & Cys (GCA)-3.R37 & TGGAGGCGCGTTCCGGAGTCGAACCGGACTAGACGGA \\
\hline & Gln2 (CTG)-3.R38 & TGGCTGGGGTACGAGGATTCGAACCTCGGAATGCCGGA \\
\hline ¿ & Glu (TTC->CTC)-3.R37 & TGGCGTCCCCTAGGGGATTCGAACCCCTGTTACCGCC \\
\hline 莣 & Gly3 (GCC)-3.R38 & TGGAGCGGGAAACGAGACTCGAACTCGCGACCCCGACC \\
\hline$\stackrel{0}{\circ}$ & His (GTG)-3.R38 & TGGGGTGGCTAATGGGATTCGAACCCACGACAACTGGA \\
\hline 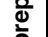 & |lle 1G72C(GAT)-3.R38 & TGGTGGGCCTGAGTGGACTTGAACCACCGACCTCACCC \\
\hline 2 & Leu2 (GAG)-3.R48 & TGGTACCGAGGACGGGACTTGAACCCGTAAGCCCTATTGGGCACTACC \\
\hline 능 & Lys (TTT->CTT)-3.R38 & |TGGTGGGTCGTGCAGGATTCGAACCTGCGACCAATTGA \\
\hline 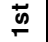 & Met (CAT)-3.R38 & TGGTGGCTACGACGGGATTCGAACCTGTGACCCCATCA \\
\hline$\stackrel{2}{\frac{2}{\pi}}$ & Phe 32C (GAA)-3.R38 & TGGTGCCCGGACTCGGAATCGAACCAAGGACACGGGGA \\
\hline 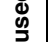 & Pro2 (GGG)-3.R38 & TGGTCGGCACGAGAGGATTTGAACCTCCGACCCCCGAC \\
\hline$\frac{0}{2}$ & Ser2 (CGA)-3.R51 & TGGCGGAGAGAGGGGGATTTGAACCCCCGGTAGAGTTGCCCCTACTCCGGT \\
\hline 玹 & Ser5 (GGA)-3.R49 & TGGCGGTGAGGGGGGGATTCGAACCCCCGATACGTTGCCGTATACACAC \\
\hline 心 & Thr3 (GGT)-3.R38 & TGGTGCTGATAGGCAGATTCGAACTGCCGACCTCACCC \\
\hline ఫָ & $\operatorname{Trp}(\mathrm{CCA})-\mathbf{3 . R 3 8}$ & TGGCAGGGGCGGAGAGACTCGAACTCCCAACACCCGGT \\
\hline & |Tyr (GTA)-3.R46 & TGGTGGTGGGGGAAGGATTCGAACCTTCGAAGTCTGTGACGGCAGA \\
\hline & Val2 (GAC)-3.R38 & TGGTGCGTCCGAGTGGACTCGAACCACCGACCCCCACC \\
\hline & nAla2L (GGC->GAG)atUUAA.R43 & GAACCGCTGACCTCTTGCTTCTCAAGCAAGCGCTCTCCCAGCT \\
\hline & nAla1L (UGC->GAG)at.R43 & GAACCGCAGACCTCCTGCACCTCAAGCAGGCGCTCTCCCAGCT \\
\hline & Ala1 (UGC)-3.R38 & TGGTGGAGCTATGCGGGATCGAACCGCAGACCTCCTGC \\
\hline & nSer2A (CGA->GGC)at.R43 UUAU & AGTTGCCCCTACTCCGGTATGCCAAACCGGTCCGTTCAGCCGC \\
\hline & nSer5A (GGA->GGC)at.R43 & ACGTTGCCGTATACACACATGCCATGCGTGCTCCTTCAGCCAC \\
\hline & fMet 1G (CAT)-3.R20 & TGGTTGCGGGGGCCGGATTT \\
\hline & Ala2 (GGC)-3.R20 & TGGTGGAGCTAAGCGGGATC \\
\hline & Arg2 (ACG->GCG)-3.R20 & |TGGTGCATCCGGGAGGATTC \\
\hline & Asn 1G72C (GTT)-3.R20 & |TGGCGCCTCTGACTGGACTC \\
\hline & Asp (GTC)-3.R20 & TGGCGGAACGGACGGGACTC \\
\hline 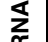 & Cys (GCA)-3.R20 & TGGAGGCGCGTTCCGGAGTC \\
\hline 荒 & Gln2 (CTG)-3.R20 & TGGCTGGGGTACGAGGATTC \\
\hline$\frac{0}{\frac{1}{2}}$ & Glu (TTC->CTC)-3.R20 & TGGCGTCCCCTAGGGGATTC \\
\hline$\frac{0}{0}$ & Gly3 (GCC)-3.R20 & TGGAGCGGGAAACGAGACTC \\
\hline$\stackrel{2}{2}$ & His (GTG)-3.R20 & |TGGGGTGGCTAATGGGATTC \\
\hline ভ্র & Ile 1G72C(GAT)-3.R20 & TGGTGGGCCTGAGTGGACTT \\
\hline ลั & Leu2 (GAG)-3.R20 & |TGGTACCGAGGACGGGACTT \\
\hline$\stackrel{ \pm}{\longleftarrow}$ & Lys (TTT->CTT)-3.R20 & TGGTGGGTCGTGCAGGATTC \\
\hline 岕 & Met (CAT)-3.R20 & TGGTGGCTACGACGGGATTC \\
\hline$\frac{n}{6}$ & Phe 32C (GAA)-3.R20 & TGGTGCCCGGACTCGGAATC \\
\hline 玹 & Pro2 (GGG)-3.R20 & TGGTCGGCACGAGAGGATTT \\
\hline$\stackrel{\$}{\mathscr{2}}$ & Ser2 (CGA)-3.R20 & TGGCGGAGAGAGGGGGATTT \\
\hline 苟 & Ser5 (GGA)-3.R20 & TGGCGGTGAGGGGGGGATTC \\
\hline & Thr3 (GGT)-3.R20 & TGGTGCTGATAGGCAGATTC \\
\hline & $\operatorname{Trp}(\mathrm{CCA})-3 . \mathrm{R} 20$ & TGGCAGGGGCGGAGAGACTC \\
\hline & Tyr (GTA)-3.R20 & TGGTGGTGGGGGAAGGATTC \\
\hline & Val2 (GAC)-3.R20 & TGGTGCGTCCGAGTGGACTC \\
\hline & Ala1 (AGC)-3.R20 & TGGTGGAGCTATGCGGGATC \\
\hline
\end{tabular}


Supplementary Table 3 (continued)

\begin{tabular}{|c|c|c|}
\hline \multirow{20}{*}{ 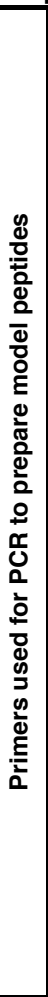 } & Y3xDDRD_gcuAla.R44 & CGAAG CTTAG TCACG GTCGT C AGC GTAGT AGTAC ATGTT TTTCT \\
\hline & Y3xDDRD_cguArg.R44 & CGAAG CTTAG TCACG GTCGT C ACG GTAGT AGTAC ATGTT TTTCT \\
\hline & Y3xDDRD_aauAsn.R44 & CGAAG CTTAG TCACG GTCGT C ATT GTAGT AGTAC ATGTT TTTCT \\
\hline & Y3xDDRD_gauAsp.R44 & CGAAG CTTAG TCACG GTCGT C ATC GTAGT AGTAC ATGTT TTTCT \\
\hline & Y3xDDRD_uguCys.R44 & CGAAG CTTAG TCACG GTCGT C ACA GTAGT AGTAC ATGTT TTTCT \\
\hline & Y3xDDRD_cagGIn.R44 & CGAAG CTTAG TCACG GTCGT C CTG GTAGT AGTAC ATGTT TTTCT \\
\hline & Y3xDDRD_gagGlu.R44 & CGAAG CTTAG TCACG GTCGT C CTC GTAGT AGTAC ATGTT TTTCT \\
\hline & Y3xDDRD_gguGly.R44 & CGAAG CTTAG TCACG GTCGT C ACC GTAGT AGTAC ATGTT TTTCT \\
\hline & Y3xDDRD_cauHis.R44 & CGAAG CTTAG TCACG GTCGT C ATG GTAGT AGTAC ATGTT TTTCT \\
\hline & Y3xDDRD_auulle.R44 & CGAAG CTTAG TCACG GTCGT C AAT GTAGT AGTAC ATGTT TTTCT \\
\hline & Y3xDDRD_cuuLeu.R44 & CGAAG CTTAG TCACG GTCGT C AAG GTAGT AGTAC ATGTT TTTCT \\
\hline & Y3xDDRD_aagLys.R44 & CGAAG CTTAG TCACG GTCGT C CTT GTAGT AGTAC ATGTT TTTCT \\
\hline & Y3xDDRD_augMet.R44 & CGAAG CTTAG TCACG GTCGT C CAT GTAGT AGTAC ATGTT TTTCT \\
\hline & Y3xDDRD_uuuPhe.R44 & CGAAG CTTAG TCACG GTCGT C AAA GTAGT AGTAC ATGTT TTTCT \\
\hline & Y3xDDRD_ccuPro.R44 & CGAAG CTTAG TCACG GTCGT C AGG GTAGT AGTAC ATGTT TTTCT \\
\hline & Y3xDDRD_ucuSer.R44 & CGAAG CTTAG TCACG GTCGT C AGA GTAGT AGTAC ATGTT TTTCT \\
\hline & Y3xDDRD_acuThr.R44 & CGAAG CTTAG TCACG GTCGT C AGT GTAGT AGTAC ATGTT TTTCT \\
\hline & Y3XDDRD_uggTrp.R44 & CGAAG CTTAG TCACG GTCGT C CCA GTAGT AGTAC ATGTT TTTCT \\
\hline & Y3xDDRD_uauTyr.R44 & CGAAG CTTAG TCACG GTCGT C ATA GTAGT AGTAC ATGTT TTTCT \\
\hline & Y3xDDRD_guuVal.R44 & CGAAG CTTAG TCACG GTCGT C AAC GTAGT AGTAC ATGTT TTTCT \\
\hline \multirow{10}{*}{ 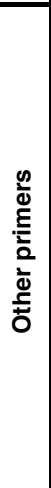 } & T7Aex.F24 & GGCGTAATACGACTCACTATAGGA \\
\hline & T7pro-leader.F36 & GTAATACGACTCACTATAGGAACGCGCGACTCTAAT \\
\hline & GFPuv.R30 & TGACTAAGAATCCAGTATGCTTACTTGTAG \\
\hline & T7g10.F26 & CTAGTAATACGACTCACTATAGGGTT \\
\hline & StvWT.R20 & ACATAGTTACTGCTGGACGG \\
\hline & StvSL.R20 & ACATAGTTACTGCTGAACGG \\
\hline & StvSLA.R20 & ACATAGTTACTGCTGGACAA \\
\hline & T7ex5.F22 & GGCGTAATACG ACTCACTATAG \\
\hline & T7esD6MYYY.F55 & TAATACGACTCACTATAGGGTTAACTTTAACAAGGAGAAAAACATGTACTACTAC \\
\hline & DRDuaaAS.R20 & TGCTGGCACTGATTCGAAGC \\
\hline
\end{tabular}

a Primer sequences used to prepare T7-tRNA templates and the DNA templates of mRNAs encoding model peptides and model proteins. 